\title{
Electronic properties of the residual donor in unintentionally doped beta-Ga2O3
}

Son Tien Nguyen, K. Goto, K. Nomura, Q. T. Thieu, R. Togashi, H. Murakami, Y. Kumagai, A. Kuramata, M. Higashiwaki, A. Koukitu, S. Yamakoshi, Bo Monemar and Erik Janzén

\section{Journal Article}

\section{Tweet}

N.B.: When citing this work, cite the original article.

Original Publication:

Son Tien Nguyen, K. Goto, K. Nomura, Q. T. Thieu, R. Togashi, H. Murakami, Y. Kumagai, A. Kuramata, M. Higashiwaki, A. Koukitu, S. Yamakoshi, Bo Monemar and Erik Janzén, Electronic properties of the residual donor in unintentionally doped beta-Ga2O3, Journal of Applied Physics, 2016. 120(23), pp..

http://dx.doi.org/10.1063/1.4972040

Copyright: AIP Publishing

http://www.aip.org/

Postprint available at: Linköping University Electronic Press

http://urn.kb.se/resolve?urn=urn:nbn:se:liu:diva-134498

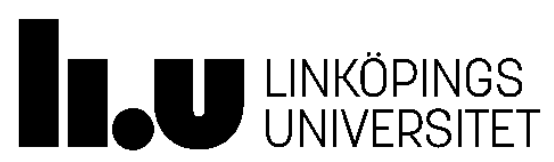




\section{Electronic properties of the residual donor in unintentionally doped $\beta-\mathrm{Ga}_{2} \mathrm{O}_{3}$}

N.T. Son ${ }^{1}$, K. Goto ${ }^{2,3}$, K. Nomura ${ }^{2}$, Q. T. Thieu², R. Togashi ${ }^{2}$, H. Murakami², Y. Kumagai², A. Kuramata ${ }^{3}$, M. Higashiwaki ${ }^{4}$, A. Koukitu², S. Yamakoshi ${ }^{3}$, B. Monemar ${ }^{1,2}$ and E. Janzén ${ }^{1}$ ${ }^{1}$ Department of Physics, Chemistry and Biology, Linköping University, SE-58183 Linköping, Sweden

${ }^{2}$ Tokyo University of Agriculture and Technology, Koganei, Tokyo 184-8588, Japan

${ }^{3}$ Tamura Corporation, Sayama, Saitama 350-1328, Japan

${ }^{4}$ National Institute of Information and Communications Technology, Koganei, Tokyo 1848795, Japan

\section{Abstract}

Electron paramagnetic resonance (EPR) was used to study the donor that is responsible for the n-type conductivity in unintentionally doped (UID) $\beta-\mathrm{Ga}_{2} \mathrm{O}_{3}$ substrates. We show that in asgrown material, the donor requires high temperature annealing to be activated. In partly activated material with the donor concentration in the $10^{16} \mathrm{~cm}^{-3}$ range or lower, the donor is found to behave as a negative-U center (often called a DX center) with the negative charge state $\mathrm{DX}^{-}$lying 16-20 meV below the neutral charge state $\mathrm{d}^{0}$ (or $\mathrm{E}_{\mathrm{d}}$ ), which is estimated to be $\sim 28-29$ meV below the conduction band minimum. This corresponds to a donor activation energy of $\mathrm{E}_{\mathrm{a}} \sim 44-49 \mathrm{meV}$. In fully activated material with the donor spin density close to $1 \times 10^{18} \mathrm{~cm}^{-3}$, donor electrons become delocalized, leading to the formation of impurity bands, which reduces the donor activation energy to $\mathrm{E}_{\mathrm{a}} \sim 15-17 \mathrm{meV}$. The results clarify the electronic structure of the dominant donor in UID $\beta-\mathrm{Ga}_{2} \mathrm{O}_{3}$ and explain the large variation in the previously reported donor activation energy.

PASC number(s): 71.55.Ht, 76.30.Da, 76.30.Pk 


\section{INTRODUCTION}

With a very large bandgap (4.8-4.9 eV) and the possibility of high $n$-type doping, gallium oxide, $\beta-\mathrm{Ga}_{2} \mathrm{O}_{3}$, is a great transparent conducting material to be used in optical devices operating in the deep ultraviolet spectral region. ${ }^{1-3}$ Its material properties are especially suited for high-voltage and high-power device applications and then even expected to be better than SiC and GaN. ${ }^{4}$ The availability of native low-cost substrates is also an advantage of $\beta-\mathrm{Ga}_{2} \mathrm{O}_{3}$ over other wide bandgap semiconductors. The metal-semiconductor field-effect transistors (MESFETs), ${ }^{4,5}$ metal-oxide-semiconductor FETs (MOSFETs) ${ }^{6}$ and Schottky barrier diodes (SBDs) have recently been demonstrated..$^{5,7,8}$

Theoretical calculations predicted that substitutional Si, Ge, Sn, F, and Cl impurities and $\mathrm{H}$ (in both substitutional and interstitial sites) have low formation energies and act as shallow donors in $\beta$-Ga2 $\mathrm{O}_{3} .{ }^{9}$ Successful $n$-doping studies have so far been reported for $\mathrm{Sn}^{7,10,11}$ and $\mathrm{Si}^{12,13}$ where electron densities in the range of $10^{16}-10^{19} \mathrm{~cm}^{-3}$ can be achieved. However, little is known on the electronic structure of shallow donors in the material. The near band-edge photoluminescence (PL) from donor-bound excitons, that can provide detailed information on the electronic structure of the involved donor in a semiconductor, has so far not been detected in $\beta-\mathrm{Ga}_{2} \mathrm{O}_{3}$.

Unintentionally doped (UID) $\beta-\mathrm{Ga}_{2} \mathrm{O}_{3}$ substrates can be highly resistive or conductive with the $n$-type conductivity. In conductive $\beta-\mathrm{Ga}_{2} \mathrm{O}_{3}$, Binet and co-workers ${ }^{14}$ observed a narrow EPR line with slightly anisotropic g-values and attributed it to the resonance of conduction electrons originating from the $\mathrm{O}$ vacancy $\left(\mathrm{V}_{\mathrm{o}}\right)$. Using highly conducting materials with the unpaired spin concentration of $10^{18}-10^{19} \mathrm{~cm}^{-3}$ this EPR signal could be detected at temperatures in the range from $4 \mathrm{~K}$ to room temperature. ${ }^{15,16}$ Mayer and co-workers ${ }^{17}$ observed that in resistive materials, the EPR signal can only be detected at elevated 
temperatures with the corresponding concentration being increased with increasing temperature. They, therefore, assigned this signal to the resonance of conduction electrons and the broad EPR signal observed at low temperatures in conducting samples to the shallow donor. Later EPR studies suggested that this donor is related to the $\mathrm{O}$ vacancy ${ }^{18}$ or the Frenkel pair of $\mathrm{V}_{\mathrm{O}}$ and the $\mathrm{O}$ interstitial, $\mathrm{V}_{\mathrm{O}}-\mathrm{O}_{\mathrm{i}} \cdot{ }^{19}$ Hall-effect measurements gave an estimated donor ionization energy in the range of $16-30 \mathrm{meV}$ for conducting $\beta-\mathrm{Ga}_{2} \mathrm{O}_{3} \cdot{ }^{20,21}$ However, hybrid functional calculations suggested $V_{0}$ to be deep donors and, therefore, cannot be responsible for the $n$-type conductivity in UID materials. ${ }^{9}$ Studies of UID $\beta-\mathrm{Ga}_{2} \mathrm{O}_{3}$ with the conductivity varying in a large range suggested that the $\mathrm{Si}$ impurity in the source material $\left(\beta-\mathrm{Ga}_{2} \mathrm{O}_{3}\right.$ powder) can account for the observed conductivity. ${ }^{21}$ The origin of the residual donor in UID material can have a strong impact on $n$-type doping of $\beta-\mathrm{Ga}_{2} \mathrm{O}_{3}$, since $\mathrm{V}_{\mathrm{O}}$-related donors are expected to be less thermally stable than substitutional impurity donors and it can be more difficult to control the conductivity using an intrinsic donor. Understanding the electronic structure and clarifying the origin of the residual donor in UID $\beta-\mathrm{Ga}_{2} \mathrm{O}_{3}$ is of technological importance and also of interest from fundamental defect physics.

In this study, we use EPR to study UID $\beta-\mathrm{Ga}_{2} \mathrm{O}_{3}$ substrates before and after hightemperature annealing for activation of the donor. We show that in partly activated materials with the spin density in the mid $10^{16} \mathrm{~cm}^{-3}$ ranges or below, the donor behaves as a negative- $\mathrm{U}$ (or DX) center, similar to the Si donor in high-Al-content AlGaN, ${ }^{22}$ while in fully activated samples, donor electrons become delocalized and the transformation from isolated donor states to corresponding impurity bands occurs, reducing the donor activation energy. In Section III. A, the DX behaviors and the activation energy of the donor is described. The delocalization of donor electrons and the decrease of the donor activation energy caused by the formation of impurity bands are presented in Section III. B, while the origin of the donor is discussed in Section III. C. 


\section{EXPERIMENTAL DETAILS}

The material used in this study is UID $\beta-\mathrm{Ga}_{2} \mathrm{O}_{3}$ (001) substrates grown by the "edge-defined film-fed growth (EFG)” technique using $\mathrm{Ga}_{2} \mathrm{O}_{3}$ powder of $5 \mathrm{~N}$ grade purity (99.999\%) with the main contaminant being Si. The melted material is pulled by a seed crystal through a capillary die made of iridium with a growth rate of $15 \mathrm{~mm} / \mathrm{h}$. The ambient of the growth reactor is a mixed gas of nitrogen (98\%) and oxygen (2\%). Details of the EFG growth are described elsewhere. ${ }^{23}$ The typical substrate size is $20 \mathrm{~mm} \times 10 \mathrm{~mm} \times 0.65 \mathrm{~mm}$. The concentration of different impurities was measured by secondary ion mass spectrometry (SIMS) at the center and near the edge (8 $\mathrm{mm}$ from the center) of the sample and the data for four impurities with high concentrations or known to act as compensating centers (Fe, $\mathrm{Mg}$ ) or donors (Si, Sn) are given in Table I. According to SIMS data, the atomic concentration of Si is at least one order of magnitude higher than that of other measured impurities. The UID samples used in this study were cleaved from (001) substrates, close to the edge. Si-doped $\beta$ $\mathrm{Ga}_{2} \mathrm{O}_{3}(-201)$ samples with the Si concentration of $\sim 3-4 \times 10^{18} \mathrm{~cm}^{-3}$, as determined from capacitance-versus-voltage (CV) measurements, were also used to study the formation of the impurity band. For partly and fully activation of the donor, the samples were annealed in $\mathrm{N}_{2}$ gas flow at $1000-1110^{\circ} \mathrm{C}$ and $1150{ }^{\circ} \mathrm{C}$, respectively.

EPR measurements were performed on an X-band ( 9.4 GHz) E500 Bruker spectrometer equipped with a continuous He-flow cryostat, allowing the regulation of the sample temperature from $4 \mathrm{~K}$ to room temperature. The concentration of the donor at the neutral charge state or the number of spins was determined from double integration of the EPR signal and measurement conditions using the spin counting application provided and calibrated by Bruker. In photo-excitation EPR (photo-EPR) experiments, a $250 \mathrm{~W}$ halogen lamp or a $150 \mathrm{~W}$ xenon lamp (for above band gap excitation) were used as excitation sources.

\section{RESULTS AND DISCUSSION}




\section{A. Negative-U behavior of the donor}

As-grown UID $\beta-\mathrm{Ga}_{2} \mathrm{O}_{3}$ substrates are of high resistivity judging from the high Q-factors $(\sim 10000-10200$ for a sample with a size of $\sim 10 \mathrm{~mm} \times 2.85 \mathrm{~mm} \times 0.65 \mathrm{~mm})$ at room temperature. The EPR spectra measured in such a sample at $297 \mathrm{~K}$ for the magnetic field $\mathbf{B}$ parallel to the $<001>$ direction in darkness and under illumination of a xenon lamp are shown in Figs. 1(a) and 1(b), respectively. As can be seen in the figure, only weak EPR signals of the $\mathrm{Fe}^{3+}$ center and an unidentified line at $\sim 3765 \mathrm{G}$ were observed. No other signals appear at lower temperatures.

Annealing at below $1000{ }^{\circ} \mathrm{C}$ for 10 minutes did not show any change in the Q-factor at room temperature. After annealing at $1100{ }^{\circ} \mathrm{C}$ for 20 minutes, the Q-factor at $293 \mathrm{~K}$ was reduced to $~ 8800$ and a very narrow EPR line with a linewidth of slightly below $0.3 \mathrm{G}$ (the magnetic field distance between the maximum and minimum of the first derivative EPR line) appeared [Fig. 1(c)]. From the angular dependence study at room temperature the g-values were determined as: $\mathrm{g}_{\mathrm{xx}}=1.9606, \mathrm{~g}_{\mathrm{yy}}=1.9577, \mathrm{~g}_{\mathrm{zz}}=1.9630$ with the principal $\mathrm{g}_{\mathrm{z}}$-axis parallel to the b-axis of the crystal and the $g_{x}$-axis is at an angle $\alpha \sim 23^{\circ}$ from the $<001>$ direction. Within the experimental errors, the g-tensor of this EPR signal is identical to that of the signal previously attributed to the resonance of conduction electrons ${ }^{14}$ and assigned later to the shallow donor related to $\mathrm{V}_{\mathrm{O}}\left(\mathrm{g}_{\mathrm{xx}}=1.960, \mathrm{~g}_{\mathrm{yy}}=1.958, \mathrm{~g}_{\mathrm{zz}}=1.962\right.$ and $\alpha \sim 24^{\circ}$ from the $<001>$ direction) ${ }^{18}$ However, the signal can only be detected at elevated temperatures ( $\left.\mathrm{T}>60 \mathrm{~K}\right)$. The spin density at the donor estimated from EPR at room temperature is $\sim 1 \times 10^{15} \mathrm{~cm}^{-3}$. In such a resistive material and a low donor concentration, it is unlikely that the resonance is related to delocalized electrons.

We have noticed that the Q-factor was hardly improved when the temperature was lowered to $\sim 50 \mathrm{~K}$ when the donor signal has already completely disappeared. This suggests that the resonance may be not related to conduction electrons, otherwise the Q-factor should 
increase at low temperatures when the density of conduction electrons is reduced. Mayer and co-workers ${ }^{17}$ also reported that in samples with high resistivity, the donor signal could only be detected at elevated temperatures. They observed that the EPR intensity increases with increasing temperature and therefore assigned again this donor signal to the resonance of conduction electrons. However, the linewidth of $2 \mathrm{G}$ at room temperature ( $~ 9$ times larger than its real linewidth) in their experiments ${ }^{17}$, indicates a huge line broadening probably due to using too high field modulations. This will lead to an overestimation of the EPR intensity at temperature ranges close to room temperature and, hence, an artificial increase of the estimated donor concentration. (In our experiments, the linewidth is $\sim 1 \mathrm{G}$ at $130 \mathrm{~K}, \sim 0.7 \mathrm{G}$ at $170 \mathrm{~K}, \sim 0.4 \mathrm{G}$ at $240 \mathrm{~K}$ and $\sim 0.22 \mathrm{G}$ at $297 \mathrm{~K}$.)

EPR spectra in this sample measured at different temperatures are shown in Fig. 2(a). As can be seen in the figure, the signal gets broader gradually when reducing the temperature and disappears at temperatures $\mathrm{T}<60 \mathrm{~K}$. The line broadening was previously attributed to a superhyperfine interaction between the electron spin and nuclear spins of nearby Ga ions. ${ }^{15,24}$ We checked the integrated intensity of the signal measured at the same temperature with different microwave powers $(0.1-0.6 \mathrm{~mW})$ and field modulation $(0.2-0.7 \mathrm{G})$ and find out that using a field modulation larger than $0.4 \mathrm{G}$ at high temperature range will over modulate the signal, which distorts and broadens the EPR line. In the temperature dependence study, the field modulation is therefore kept at $0.4 \mathrm{G}$ and the microwave power of $\sim 0.1 \mathrm{~mW}$ at high temperatures in order to avoid signal overloaded, and at $\sim 0.6 \mathrm{~mW}$ when measuring at low temperatures in order to improve the signal-to-noise ratio when the EPR signal becomes weaker.

The temperature dependence of the donor spin density (or the concentration of donors in the neutral charge state), $n(T)$, estimated from EPR using the spin-counting application is shown in Fig. 2(b). The maximum donor concentration in the neutral charge state does not 
occur at the lowest temperature as expected for a normal shallow donor but appears at an elevated temperature. Such a temperature dependence of $n(T)$ is typical for a negative-U donor, often called a DX center, similar to the behavior of the Si donor in high-Al-content $\mathrm{AlGaN}^{22}$ In a DX center, the donor in the neutral charge state $\mathrm{E}_{\mathrm{d}}$ prefers to capture a second electron to pair off the spins and undergoes a large lattice relaxation to reduce its energy. ${ }^{25} \mathrm{At}$ low temperatures, the donors are mainly in the negative charge state $\mathrm{DX}^{-}$, which lies below $E_{d}$ and is EPR inactive with the electron spin $S=0$. Depending on the energy separation between $\mathrm{E}_{\mathrm{d}}$ and $\mathrm{DX}^{-}$(or $\mathrm{E}_{\mathrm{DX}}$ ), the signal can be measured at elevated temperatures when the thermally induced population on $E_{d}$ becomes detectable by EPR. With increasing temperature, the spin density at $\mathrm{E}_{\mathrm{d}}, \mathrm{n}(\mathrm{T})$, keeps increasing until the temperature is high enough to excite electrons from $E_{d}$ to the first effective-mass excited state $2 p$ [see the energy scheme in the inset of Fig. 2(b)]. Due to competition between these two processes, n(T) will reach a maximum and then reduces with increasing temperature when the process of removing electrons from $E_{d}$ to $E_{2 p}$ becomes dominating. With considering only $E_{D x}, E_{d}$ and the first effective-mass excited state $2 p$, the donor spin density $n(T)$ can be described as ${ }^{22}$

$$
\mathrm{n}(\mathrm{T}) \propto \frac{\mathrm{N}}{1+\mathrm{e}^{\left(\mathrm{E}_{\mathrm{d}}-\mathrm{E}_{\mathrm{F}}\right) / \mathrm{k}_{\mathrm{B}} \mathrm{T}}+\mathrm{Ce}^{-\left(\mathrm{E}_{2 \mathrm{p}}-\mathrm{E}_{\mathrm{d}}\right) / \mathrm{k}_{\mathrm{B}} \mathrm{T}} .}
$$

Here $\mathrm{N}$ is the total donor concentration, $\mathrm{k}_{\mathrm{B}}$ is the Boltzmann constant, $\mathrm{E}_{\mathrm{F}}$ is the Fermi level with the energy reference at the conduction band minimum $(\mathrm{CBM}), \mathrm{E}_{\mathrm{C}}=0$, and $\mathrm{C}$ is a factor taking into account the degeneracy of higher-lying excited states within $\mathrm{k}_{\mathrm{B}} \mathrm{T}$ from the 2p state. From the best fit to $n(T)$ we observed $E_{d}-E_{F}=10 \mathrm{meV}$ and $E_{2 p}-E_{d}=22 \mathrm{meV}$. Assuming that the neutral charge state $E_{d}$ and excited states of the donor follow the effective mass theory, i.e. the $E_{d} / i^{2}$ rule $(i=1,2, . ., n)$ or $E_{2 p} \sim E_{d} / 4$ and $\left|E_{2 p}-E_{d}\right|=\left|\left(E_{d} / 4\right)-E_{d}\right|=3\left|E_{d}\right| / 4$, we can estimate $\left|E_{d}\right|=4\left|E_{2 p}-E_{d}\right| / 3 \sim 29 \mathrm{meV}$. For a negative-U center, the Fermi level lies at the middle between the ground state occupied state ${ }^{26}$ In the case of a DX donor, EF lies close to the middle between $\mathrm{E}_{\mathrm{d}}$ and $\mathrm{E}_{\mathrm{Dx}}$ levels and $\left|\mathrm{E}_{\mathrm{Dx}}\right| \sim\left|\mathrm{E}_{\mathrm{d}}\right|+2\left|\mathrm{E}_{\mathrm{d}}-\mathrm{E}_{\mathrm{F}}\right| \sim 29+20 \sim 49 \mathrm{meV}$. Thus, 
in resistive substrates with the donor concentration in the mid $10^{15} \mathrm{~cm}^{-3}$ range, the activation energy of the donor is expected to be $\mathrm{E}_{\mathrm{a}}=\mathrm{E}_{\mathrm{DX}} \sim 49 \mathrm{meV}$.

After annealing further the same sample at $1110^{\circ} \mathrm{C}$ in $\mathrm{N}_{2}$ gas flow for 180 minutes, we observe that the Q-factor at $293 \mathrm{~K}$ is reduced to $\sim 5400$, indicating a more conducting, but still rather resistive condition. We performed again the temperature dependence study of $n(T)$ in this sample and the result is shown in Fig. 3. Now the EPR signal can be detected down to temperatures of $\sim 40 \mathrm{~K}$ and we observe a similar temperature dependence of $\mathrm{n}(\mathrm{T})$, with its maximum slightly shifted to lower temperatures. The fit using Eq. (1) gives $E_{d}-E_{F}=8 \mathrm{meV}$, $E_{2 p}-E_{d}=21 \mathrm{meV}$ and, hence, $E_{d}=28 \mathrm{meV}$. These values are slightly smaller than that in the sample before the second annealing at $1110^{\circ} \mathrm{C}$, but the deviations are within the errors of the method. The donor activation energy is then determined as: $E_{a}=E_{d}+2\left|E_{d}-E_{F}\right|=28+16=44$ $\mathrm{meV}$. Thus, with the concentration of low $10^{16} \mathrm{~cm}^{-3}$ range as estimated by EPR at $\sim 70 \mathrm{~K}$, the donor also shows clear DX behavior.

Due to different electronic structure, a normal shallow donor and a DX donor can show different responses to illumination. Under illumination, electrons being trapped at deep levels, such as the Ga vacancies and other compensated impurities, e.g. Fe and Mg, can be excited to the neutral charge state $\mathrm{d}^{0}$ via the conduction band. This usually leads to an increase of the EPR signal of a shallow donor. However, this is not always the case for a DX donor since electrons on $\mathrm{d}^{0}$ can relax down to the negative charge state $\mathrm{DX}^{-}$if they can get enough thermal energy to overcome the barrier between the two charge states. The increase of the donor signal may be observed only at low temperatures, depending on the barrier height. An example is the Si DX donor in AlN, which shows an enhancement of the EPR signal under illumination only at temperatures below $60 \mathrm{~K} \cdot{ }^{27,28}$ We performed photo-EPR using both below and above band gap excitations and found that illumination has no noticeable effect on the EPR signal of the donor at all studied temperatures. Fig. 4 shows no changes in the 
intensity of the donor signal measured at $80 \mathrm{~K}$ in darkness and under above-band-gap excitation. At temperatures below $30 \mathrm{~K}$, the donor signal is not observable, neither in darkness nor under illumination. This suggests that the energy barrier between the neutral and negative charge states is very low and electrons on the $\mathrm{d}^{0}$ state can relax to the $\mathrm{DX}^{-}$state even at low temperatures.

\section{B. Delocalized donor electrons and formation of impurity bands}

In the sample where the donor has been activated by annealing at $1150{ }^{\circ} \mathrm{C}$, the conductivity is higher and the coupling of the microwave can be possible only at low temperatures with much smaller Q-factors ( 500-700 at below $60 \mathrm{~K})$. The EPR signal can now be detected down to 4 $\mathrm{K}$, indicating that the Fermi level in this sample locates at $\mathrm{E}_{\mathrm{d}}$ or slightly above similar to the case of an effective mass shallow donor. The donor concentration estimated at $10 \mathrm{~K}$ is $\sim 9 \times 10^{17} \mathrm{~cm}^{-3}$, which is close to the SIMS concentration of Si near the edge of UID substrates $\left(\sim 7 \times 10^{17} \mathrm{~cm}^{-3}\right)$. The temperature dependence of $\mathrm{n}(\mathrm{T})$ in this sample in the temperature range below $60 \mathrm{~K}$ is shown in Fig. 5.

It is possible that at such a high donor concentration, a slightly overlap of the wave function between donors may occur and an electron in a $\mathrm{DX}^{-}$state can tunnel to a nearby ionized donor state, $\mathrm{d}^{+}$, even at low temperatures. Unlike a normal shallow donor, whose ionized states are available in equilibrium only if compensated deep levels are present in the material, ${ }^{29}$ a DX center has an equal number of negatively charged donors and ionized donors. The tunneling of an electron from a negatively charged donor to a nearby ionized donor is therefore possible, following the process ${ }^{25}: \mathrm{DX}^{-}+\mathrm{d}^{+} \rightarrow 2 \mathrm{~d}^{0}$, which creates two neutral donors. As the result, electrons on DX donors become delocalized and their isolated donor states transform to impurity bands. This process makes it possible to observe the EPR signal of the neutral donor at low temperatures. The formation of the $\mathrm{d}^{0}$ band also effectively reduces the energy required to excite unpaired electrons to the first excited state E2p. The 
concentration $\mathrm{n}(\mathrm{T})$ of the donor in the $\mathrm{d}^{0}$ band follows the thermal distribution as the case of a shallow effective mass donor ${ }^{30,31}$

$$
\mathrm{n}(\mathrm{T}) \propto \frac{\mathrm{N}}{1+0.5 \mathrm{e}^{\left(\mathrm{E}_{\mathrm{d}}-\mathrm{E}_{\mathrm{F}}\right) / \mathrm{k}_{\mathrm{B}} \mathrm{T}}+\mathrm{Ce} \mathrm{e}^{-\left(\mathrm{E}_{2 \mathrm{p}}-\mathrm{E}_{\mathrm{d}}\right) / \mathrm{k}_{\mathrm{B}} \mathrm{T}} .}
$$

The best fit of $n(T)$ using Eq. (2) gives $E_{d}-E_{F}=-2 m e V$ and $E_{2 p}-E_{d} \sim 10 m e V$, which is about a half of the expected value (21-22 meV) for the case of a single $\mathrm{d}^{0}$ state when the impurity bands have not been formed. Taking into account the above obtained energy from the $2 p$ state to the CBM of $\left(E_{c}-E_{2 p}\right)=\left(E_{2 p}-E_{d}\right) / 3 \sim 21 / 3 \sim 7 \mathrm{meV}$, the donor activation energy is expected to be $\mathrm{E}_{\mathrm{a}} \sim 10+7=17 \mathrm{meV}$ for $\mathrm{UID} \beta-\mathrm{Ga}_{2} \mathrm{O}_{3}$ after full activation of the donor.

The formation of impurity bands with delocalized electrons causes the skin effect, which hinders the penetration of the microwaves, resulting in the typical asymmetric Dysonian line shape ${ }^{32,33}$ of the EPR signal as seen in the case of highly Si-doped GaN. ${ }^{34}$ In a normal shallow donor, the negatively charged donor band $\mathrm{D}^{-}$lies above the $\mathrm{d}^{0}$ band, just below the CBM, ${ }^{29}$ while in a DX donor as in our case, the highest occupied band is the $\mathrm{d}^{0}$ band. Thus, electrons, that give rise to the observed EPR signal, are still related to the donor although they can tunnel from a donor to another donor. Therefore, the g-values of the EPR spectrum should represent the properties of the donor and not electrons in the conduction band.

We measured again the angular dependences of the g-value in the partly activated sample (after annealing at $1110{ }^{\circ} \mathrm{C}$ ) at two different temperatures: $80 \mathrm{~K}$ and $292 \mathrm{~K}$. Their corresponding g-values versus the angle of the magnetic field in the (010) plane are shown in Fig. 6(a). The angular dependence measured at $80 \mathrm{~K}$ shows the same principal g-values (except the $g_{y y}$-component being 0.0002 smaller) and the angles of principal axes and as compared to that measured at $292 \mathrm{~K}$. These g-values are also the same as the g-values of the 
donor in the sample annealing at $1100{ }^{\circ} \mathrm{C}$. Figure 6(b) shows the angular dependence of the gvalue in the fully activated sample (after annealing and $1150^{\circ} \mathrm{C}$ ) measured at $15 \mathrm{~K}$ with the magnetic field rotating from the $<010>$ direction to the $<001>$ direction. The obtained gtensor is the same as that in the partly activate sample.

In a previous study ${ }^{18}$, the g-values were found to be unchanged (within the experimental errors of $\Delta \mathrm{g}=0.0007)$ in the temperature range of 5-300 K. Our observed increase of the g-value with increasing temperature is similar to the observation in Ref. 17. Such a g-value shift is not expected for a normal shallow donor, whose g-values are usually decreases with increasing temperature. The g-value shift in this case mainly occurs for the gyy and $g_{z z}$ components, i.e. the g-value at the direction close to the normal of the cleavage plane (100) and in the $<010>$ direction of the b-axis. It is possible that this shift of the g-value with temperature is related to the changes in the local environment of the donor induced by the anisotropic thermal expansion of the crystal with increasing temperature, which was observed in the study of the lattice expansion by Víllora and co-workers. ${ }^{35}$ Thus, all the EPR studies so far show that the donor in either resistive or highly conducting $\beta-\mathrm{Ga}_{2} \mathrm{O}_{3}$ has the same anisotropic g-tensor. The anisotropic g-values in the range 1.958-1.963 are typical for a shallow donor. Delocalized electrons in the $\mathrm{D}^{-}$band of a normal shallow donor, which lies just below the conduction band, are actually free electrons in the conduction band. Binet and co-workers ${ }^{14}$ suggested that the anisotropy of the g-values of the resonance of conduction electrons is caused by the anisotropy of the conduction band structure of $\beta-\mathrm{Ga}_{2} \mathrm{O}_{3}$. In the frame of the DX model, both the delocalization of electrons and the anisotropy of the g-value can be well explained since the delocalized electrons are confined in the $\mathrm{d}^{0}$ band, not in the conduction band.

In the UID sample annealed at $1150{ }^{\circ} \mathrm{C}$ with a donor activation energy $\mathrm{E}_{\mathrm{a}} \sim 17 \mathrm{meV}$, the EPR line is symmetric, showing no Dysonian line shape (see the inset of Fig. 5). This 
means that the delocalization of electrons may occur locally and the conductivity is not high enough to induce the skin effect that causes the asymmetric resonance line shape. ${ }^{32,33}$ In another UID sample with a slightly higher donor concentration $\left(\sim 1.5 \times 10^{18} \mathrm{~cm}^{-3}\right.$ as estimated by EPR), the transition from a normal line shape to the Dysonian line shape can be detected in the temperature range $\sim 20-30 \mathrm{~K}$ [see Fig. 7(a)]. As can be seen in the figure, the EPR line measured at $20 \mathrm{~K}$ is symmetric with equal amplitude of the positive and negative parts of the line (A/B $~ 1)$. At $\sim 30 \mathrm{~K}$ and higher temperatures, this ratio increases to $\mathrm{A} / \mathrm{B} \sim 1.8-1.9$ and the line shows a clear Dysonian shape. A similar transition from Lorentzian to Dysonian line shape was also seen in the study by Mayer and co-workers at the temperature range 50-100 K [see Fig. 2(b) in Ref. 17]. In an intentionally Si-doped substrate with the donor concentration of $\sim 3-4 \times 10^{18} \mathrm{~cm}^{-3}$, the Dysonian line shape is already observed at $6.5 \mathrm{~K}$ [see Fig. 7(b)]. The ratio $\mathrm{A} / \mathrm{B} \sim 1.9$ is smaller than that of conduction electrons (the $\mathrm{A} / \mathrm{B}$ ratio varies between 2.7 and 19), ${ }^{32,33}$ suggesting that the level of electron delocalization in our study is moderate.

The linewidth narrowing with increasing temperature was previously observed and explained as due to quenching of the hyperfine interaction between the donor electron spin and the nuclear spins of surrounding nuclei caused by the motion of delocalized electrons between donor sites. ${ }^{15,17,24}$ The linewidth narrowing observed in our partly activated, highresistance samples [see Fig. 2(a)] cannot be due to the motion of delocalized electrons, but may be mainly caused by the enhancement of the average lifetime of electrons on the neutral charge state with increasing temperature since $\mathrm{d}^{0}$ is actually the excited state, not the ground state of this DX donor. The linewidth narrowing with increasing temperature due to the motion of delocalized electrons is expected in high conducting samples as can be seen in Figs. 7(a) and 7(b). In addition to the effect of temperature, linewidth narrowing is also enhanced with increasing the concentration of delocalized electrons. In the UID sample with the donor concentration of $\sim 1.5 \times 10^{18} \mathrm{~cm}^{-3}$, the linewidth at $20 \mathrm{~K}$ is $\sim 3 \mathrm{G}$ [Fig. 7(a)], while in the sample 
doped with Si to a concentration of $\sim 3-4 \times 10^{18} \mathrm{~cm}^{-3}$, the linewidth is only $\sim 1.1 \mathrm{G}$ at $18.5 \mathrm{~K}$ and $\sim 1.5 \mathrm{G}$ at $6.5 \mathrm{~K}$.

The temperature dependence of $\mathrm{n}(\mathrm{T})$ in the UID sample with the donor concentration $\sim 1.5 \times 10^{18} \mathrm{~cm}^{-3}$ is shown in Fig. 8. The best fit using Eq. (2) gives $E_{d}-E_{F}=-2 \mathrm{meV}$ and $E_{2 p}-$ $E_{d}=8 \mathrm{meV}$. This energy threshold is close to the value of $7.5 \mathrm{meV}$ reported by Mayer and co-workers ${ }^{17}$ in conducting $\beta-\mathrm{Ga}_{2} \mathrm{O}_{3}$ and of $\sim 7 \mathrm{meV}$ reported by Víllora et al ${ }^{35}$ in UID $\beta$ $\mathrm{Ga}_{2} \mathrm{O}_{3}$ samples grown by floating zone technique using less pure source materials (99.99\%) with the carrier concentration in the low $10^{18} \mathrm{~cm}^{-3}$ range. Adding the energy distance $\mathrm{E}_{\mathrm{c}}-\mathrm{E}_{2 \mathrm{p}}$ $\sim 7 \mathrm{meV}$, the donor activation energy is expected to be $\mathrm{E}_{\mathrm{a}} \sim 15 \mathrm{meV}$ for $\beta-\mathrm{Ga}_{2} \mathrm{O}_{3}$ with the donor concentration in the low $10^{18} \mathrm{~cm}^{-3}$ range.

\section{Origin of the donor}

Recent $a b$ initio calculations ${ }^{9}$ suggest that $\mathrm{V}_{\mathrm{O}}$ is a deep donor with the $(2+\mid 0)$ level located at more than $1 \mathrm{eV}$ below the CBM, and for all Fermi levels positions, $\mathrm{V}_{\mathrm{O}}^{+}$can be observable by EPR only under illumination. The $\mathrm{V}_{\mathrm{o}}$ center in $\mathrm{ZnO}$ also shows similar properties, i.e. being a deep donor with $\mathrm{V}_{\mathrm{O}}^{+}$only detectable by EPR under illumination. ${ }^{36,37}$ The donor in UID $\beta$ $\mathrm{Ga}_{2} \mathrm{O}_{3}$, with an activation energy being much smaller than that predicted for $V_{0}$ and with its signal detectable in darkness, is unlikely to be related to the O vacancy. In a recent EPR study, Yamaga and co-workers ${ }^{19}$ noticed that in $\beta-\mathrm{Ga}_{2} \mathrm{O}_{3}$ doped with $\mathrm{Si}, \mathrm{Zr}$, and Hf, an EPR line with the same g-values was observed. They suggested a defect model, in which the excess electron from the substitutional impurity being trapped at the O vacancy-O interstitial Frenkel pair, $\mathrm{V}_{\mathrm{O}-\mathrm{O}}$, to explain the EPR observation. However, our annealing studies show that the donor is very thermally stable and may not be explained by the model of the $\mathrm{V}_{\mathrm{O}}-\mathrm{O}_{\mathrm{i}}$ Frenkel pair, which is expected to be dissociated at high temperatures. It is known that Si substituting for $\mathrm{Ga}$ and $\mathrm{O}$ at the $\mathrm{N}$ site are shallow donors in GaN and their EPR spectra are not 
distinguishable in the g-values. Therefore, it is possible that $\mathrm{Si}, \mathrm{Sn}, \mathrm{Zr}$ and $\mathrm{Hf}$ donors in $\beta$ $\mathrm{Ga}_{2} \mathrm{O}_{3}$ give rise to EPR signals with similar g-values.

The donor in UID $\beta-\mathrm{Ga}_{2} \mathrm{O}_{3}$ was previously suggested to be related to Si based on its presence in the source material with the concentration similar to the free carrier concentration determined from Hall-effect measurements ${ }^{21}$ or from the Si-doping studies. ${ }^{12,13}$ A recent study also shows that the free carriers activated by annealing in $\mathrm{N}_{2}$ gas are similar to the doped $\mathrm{Si}$ concentration. ${ }^{23}$ In Si-doped $\beta-\mathrm{Ga}_{2} \mathrm{O}_{3}$ to a concentration of $1 \times 10^{19} \mathrm{~cm}^{-3}$, the free carrier concentration was found to increase sharply with annealing at $800-1000{ }^{\circ} \mathrm{C}$ and reaches an electrically activated level of more than $80 \% .^{12}$ In Si-doping of $\beta-\mathrm{Ga}_{2} \mathrm{O}_{3}$ epitaxial layers grown by halide vapor phase epitaxy, a controlled n-type conductivity with the carrier concentration from $10^{15}$ to $10^{18} \mathrm{~cm}^{-3}$ could be achieved and post annealing in $\mathrm{N}_{2}$ gas at $\sim 1000$ ${ }^{\circ} \mathrm{C}$ for activation of the Si donor is a part of the doping procedure. ${ }^{38} \mathrm{~A}$ recent study of Sidoping in $\beta-\mathrm{Ga}_{2} \mathrm{O}_{3}$ epitaxial layers grown by metal organic vapor phase epitaxy reported that with the Si concentration up to $4 \times 10^{18} \mathrm{~cm}^{-3}$, the layer is still semi-insulating after annealing at 800-850 ${ }^{\circ} \mathrm{C}$ in $\mathrm{O}_{2}$ gas for one hour. ${ }^{39}$ Kuramata and co-workers ${ }^{23}$ also showed that in the same set of $\beta-\mathrm{Ga}_{2} \mathrm{O}_{3}$ samples doped with $\mathrm{Si}$ to the concentration in the range of $10^{18}-10^{19} \mathrm{~cm}^{-3}$, annealing in $\mathrm{O}_{2}$ gas at $1150{ }^{\circ} \mathrm{C}$ did not activate the donors, but annealing at $800-1000{ }^{\circ} \mathrm{C}$ in $\mathrm{N}_{2}$ gas gave rise to the free carrier concentration in the same Si doping range. While it is still unclear why annealing in $\mathrm{O}_{2}$ prevents $\mathrm{Si}$-doped $\beta-\mathrm{Ga}_{2} \mathrm{O}_{3}$ becoming conductive, the reported doping studies all suggest that $\mathrm{Si}$ is the source of free electrons.

At room temperature, with the $\mathrm{DX}^{-}$state lying only $16-20$ meV below the neutral state of the effective mass donor, Si will provide free electrons as a shallow donor. As shown from SIMS, Si is the dominant impurity with the concentration comparable to the donor concentration estimated by EPR. Thus, Si should have the dominant contribution to the EPR signal of the donor in UID and activated $\beta-\mathrm{Ga}_{2} \mathrm{O}_{3}$. Considering the requirement of high 
temperature annealing for donor activation and the fair correlation between the concentration of donor determined by EPR and the concentration of Si obtained from SIMS, Si appears to be the best candidate for the donor that is responsible for the residual n-type conductivity in UID $\beta-\mathrm{Ga}_{2} \mathrm{O}_{3}$. The requirement of high-temperature annealing for donor activation may suggest that $\mathrm{Si}$ after the growth is passivated by other impurities or intrinsic defects, similar to the case of Mg acceptors in GaN. ${ }^{40}$ Unfortunately, the DX structure with the neutral state $\mathrm{d}^{0}$ lying above the ground state $\mathrm{DX}^{-}$makes it impossible to saturate the EPR transition even using the maximum available microwave power of $200 \mathrm{~mW}$. Therefore, electron nuclear double resonance experiments cannot be performed for chemical identification of the donor.

\section{SUMMARY}

In summary, using EPR we study UID $\beta-\mathrm{Ga}_{2} \mathrm{O}_{3}$ substrates before and after annealing in $\mathrm{N}_{2}$ gas ambient for activation of the donor at different levels corresponding to different donor concentrations and conductivities. We show that as-grown material is highly resistive, showing no EPR signal of the donor. After annealing at $\sim 1100-1110{ }^{\circ} \mathrm{C}$ the donor is partly activated with the donor spin density in the range of mid $10^{15}$ - low $10^{16} \mathrm{~cm}^{-3}$ and shown to behaves as a negative- $\mathrm{U}$ center with the negative charge state $\mathrm{DX}^{-}$locating at $\sim 16-20 \mathrm{meV}$ below the neutral charge state $\mathrm{E}_{\mathrm{d}}$, which lies at $28-29 \mathrm{meV}$ below the CBM. The corresponding donor activation energy is estimated to be $\mathrm{E}_{\mathrm{a}} \sim 44-49 \mathrm{meV}$. After annealing at $1150{ }^{\circ} \mathrm{C}$, the donor is fully activated with the concentration $\sim 9 \times 10^{17} \mathrm{~cm}^{-3}$ and donor electrons become partly delocalized, forming impurity bands, which reduces the donor activation energy to $\mathrm{E}_{\mathrm{a}} \sim 17 \mathrm{meV}$. In UID samples with the donor concentration reaching $\sim 1 \times 10^{18} \mathrm{~cm}^{-3}$ or in Si-doped samples with the concentration of $\sim 3-4 \times 10^{18} \mathrm{~cm}^{-3}$, the EPR signal shows a clear Dysonian line shape, which is typical for the resonance of delocalized electrons, and a donor activation energy of $\sim 15 \mathrm{meV}$ is determined. The $\mathrm{DX}$ properties of the donor and the formation of impurity bands explain the large variation of the donor activation energies 
previously reported by transport measurements. Based on the correlation between the donor concentration estimated by EPR and the Si concentration determined by SIMS as well as their annealing behaviors, the donor responsible for the $n$-type conductivity in $\mathrm{UID} \beta-\mathrm{Ga}_{2} \mathrm{O}_{3}$ is assigned to Si. Due to a small energy distance between the $\mathrm{DX}^{-}$state and the neutral state $\mathrm{d}^{0}$ of $\sim 20 \mathrm{meV}$, Si will behave as a shallow donor at the operating temperature of devices.

\section{Acknowledgments}

Support from the Linköping Linnaeus Initiative for Novel Functional Materials (LiLi-NFM), the Council for Science, Technology and Innovation (CSTI), Cross-ministerial Strategic Innovation Promotion Program (SIP), “Next-generation power electronics” (funding agency: NEDO, Japan) is acknowledged.

\section{References}

${ }^{1}$ K. Shimamura, E. G. Víllora, K. Domen, K. Yui, K. Aoki, and N. Ichinose, Jpn. J. Appl. Phys. 44, L7 (2005).

${ }^{2}$ Y. Kokubun, K. Miura, F. Endo, and S. Nakagomi, Appl. Phys. Lett. 90, 031912 (2007).

${ }^{3}$ T. Oshima, T. Okuno, N. Arai, N. Suzuki, S. Ohira, and S. Fujita, Appl. Phys. Express 1, 011202 (2008).

${ }^{4}$ M. Higashiwaki, K. Sasaki, A. Kuramata, T. Masui, and S. Yamakoshi, Appl. Phys. Lett. 100, 013504 (2012).

${ }^{5}$ M. Higashiwaki, K. Sasaki, A. Kuramata, T. Masui, and S. Yamakoshi, Phys. Status Solidi A 211, 21 (2014).

${ }^{6}$ M. Higashiwaki, K. Sasaki, T. Kamimura, M. H. Wong, D. Krishnamurthy, A. Kuramata, T. Masui, and S. Yamakoshi, Appl. Phys. Lett. 103, 123511 (2013).

${ }^{7}$ K. Sasaki, A. Kuramata, T. Masui, E. G. Víllora, K. Shimamura, and S. Yamakoshi, Appl. Phys. Express 5, 035502 (2012).

${ }^{8}$ H. Murakami, K. Nomura, K. Goto, K. Sasaki, K. Kawara, Q. T. Thieu, R. Togashi, Y. Kumagai, M. Higashiwaki, A. Kuramata, S. Yamakoshi, B. Monemar, and A. Koukitu, Appl. Phys. Express 8, 015503 (2015).

${ }^{9}$ J. B. Varley, J. R. Weber, A. Janotti, and C. G. Van de Walle, Appl. Phys. Lett. 97, 142106 (2010).

${ }^{10}$ N. Ueda, H. Hosono, R. Waseda, and H. Kawazoe, Appl. Phys. Lett. 70, 3561 (1997).

${ }^{11}$ N. Suzuki, S. Ohira, M. Tanaka, T. Sugawara, K. Nakajima, and T. Shishido, Phys. Status 
Solidi C 4, 2310 (2007).

${ }^{12}$ E. G. Víllora, K. Shimamura, Y. Yoshikawa, T. Ujiie, and K. Aoki, Appl. Phys. Lett. 92, 202120 (2008).

${ }^{13}$ K. Sasaki, M. Higashiwaki, A. Kuramata, T. Masui, and S. Yamakoshi, Appl. Phys. Express 6, 086502 (2013).

${ }^{14}$ L. Binet, D. Gourier and C. Minot, Journal of Solid State Chemistry 113, 420 (1994).

${ }^{15}$ E. Aubay and D. Gourier, Phys. Rev. B 47, 15023 (1993).

${ }^{16}$ D. Gourier, E. Aubay and J. Guglielmi, Phys. Rev. B 50, 2941 (1994).

${ }^{17}$ B. K. Meyer, U. Leib, A. Hofstaetter, C. Krummel, and D. Kohl, Mater. Sci. Forum 258263, 1473 (1997).

${ }^{18}$ M. Yamaga, E.G. Villora, K. Shimamura, N. Ichinose, M. Honda, Phys. Rev. B 68, 155207 (2003).

${ }^{19}$ M. Yamaga, H. Tsuzuki, S. Takano, E. G. Villora, K. Shimamura, J. Non-Cryst. Solids 358, 2458 (2012).

${ }^{20}$ M.R. Lorenz, J.F. Woods and R.J. Gambino, J. Phys. Chem. Solids 28, 403 (1967).

${ }^{21}$ K. Irmscher, Z. Galazka, M. Pietsch, R. Uecker, and R. Fornari, J. Appl. Phys. 110, 063720 (2011).

${ }^{22}$ X.T. Trinh, D. Nilsson, I.G. Ivanov, E. Janzén, A. Kakanakova-Georgieva, and N.T. Son, Appl. Phys. Lett. 105, 162106 (2014).

${ }^{23}$ A. Kuramata, K. Koshi , S. Watanabe, Yu Yamaoka, T. Masui, and S. Yamakoshi, J. J. Appl. Phys. 55, 1202A2 (2016).

${ }^{24}$ L. Binet and D. Gourier, J. Phys. Chem. Solids 59, 1241 (1998).

${ }^{25}$ D. J. Chadi and K. J. Chang, Phys. Rev. Lett. 61, 873 (1988). Phys. Rev. B 39, 10063 (1989).

${ }^{26}$ D. Adler and E. J. Yoffa, Phys. Rev. Lett 36, 1197 (1976).

${ }^{27}$ M. W. Bayerl, M. S. Brandt, T. Graf, O. Ambacher, J. A. Majewski, M. Stutzmann, D. J. As, and K. Lischka, Phys. Rev. B 63, 165204 (2001).

${ }^{28}$ N. T. Son, M. Bickermann, and E. Janzén, Appl. Phys. Lett. 98, 092104 (2011).

${ }^{29}$ B.I. Shklovskii and A.L. Efros, in Electronic properties of doped semiconductors (SpringerVerlag Berlin Heidelberg 1984), p. 52.

${ }^{30}$ N.W. Ashcroft and N.D. Mermin, Solid State Physics (Books/Cole, Bermont, 1976), p. 581.

${ }^{31}$ N.T. Son, Y.-S. Kim, and E. Janzén, Phys. Status Solidi RRL 9, 707 (2015), Supporting Information. 
${ }^{32}$ G. Feher and A.F. Kip, Phys. Rev. 98, 337 (1955).

${ }^{33}$ F.J. Dyson, Phys. Rev. 98, 349 (1955).

${ }^{34}$ A. Wolos, Z. Wilamowski, M. Piersa, W. Strupinski, B. Lucznik, I. Grzegory, and S. Porowski, Phys. Rev. B 83, 165206 (2011).

${ }^{35}$ E.G. Víllora, K. Shimamura, T. Ujiie, and K. Aoki, Appl. Phys. Lett. 92, 202118 (2008).

${ }^{36}$ J. M. Smith, V.H. Vehse, Phys. Lett. A 31, 147 (1970).

${ }^{37}$ C. Gonzales, D. Galland, and A. Hervé, Phys. Stat. Sol. (b) 72, 309 (1975).

${ }^{38}$ K. Goto, K. Nomura, H. Murakami, Q. T. Thieu, R. Togashi, Y. Kumagai, A. Kuramata, B. Monemar, A. Koukitu, and S. Yamakoshi, Abstract G4, International Workshop on Gallium Oxide and Related Materials, Kyoto, 2015.

${ }^{39}$ D. Gogova, G. Wagner, M. Baldini, M. Schmidbauer, K. Irmscher, R. Schewski, Z. Galazka, M. Albrecht, and R. Fornari, J. Crys. Growth 401, 665 (2014).

${ }^{40}$ S. Nakamura, T. Mukai, M. Senoh and N. Iwasa, J. J. Appl. Phys. 31, L139 (1992). 
Table I: Concentration, given in the unit of $10^{17} \mathrm{~cm}^{-3}$, of Si, Sn, Fe and Mg measured by SIMS at the center and near the edge ( $\sim 8 \mathrm{~mm}$ from the center) of the UID $\beta-\mathrm{Ga}_{2} \mathrm{O}_{3}$ substrate with the size of $10 \mathrm{~mm} \times 20 \mathrm{~mm}$,. The samples used in this study are cleaved from the region closed to the edge of the substrate.

\begin{tabular}{|c|c|c|c|c|}
\hline Position & Si & Fe & Sn & Mg \\
\hline Center & 1.1 & 0.1 & 0.05 & 0.03 \\
Edge & 7.1 & 0.4 & 0.2 & 0.8 \\
\hline
\end{tabular}




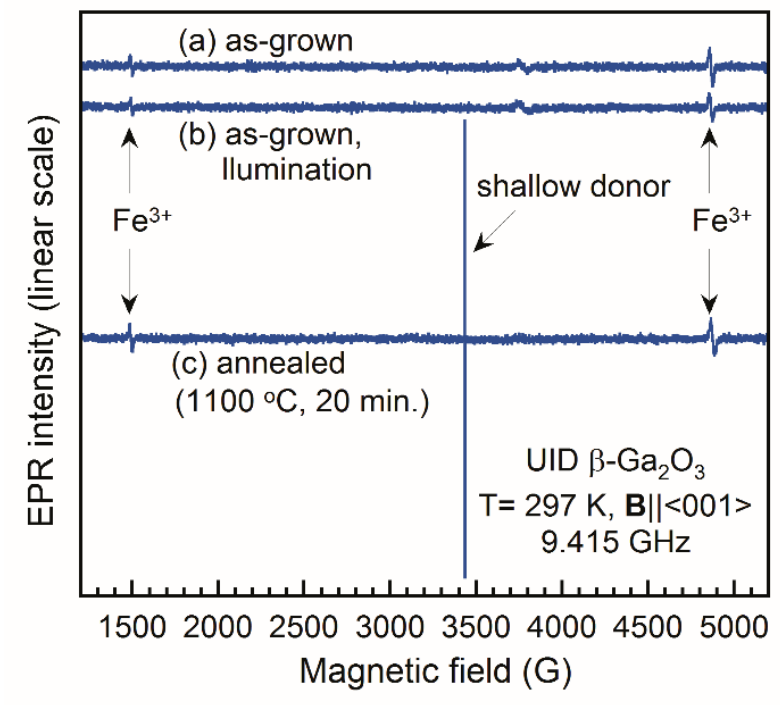

FIG1: (Color online) EPR spectra measured in an as-grown UID $\beta-\mathrm{Ga}_{2} \mathrm{O}_{3}$ substrate for $\mathbf{B} \|<001>$ (a) in darkness, and (b) under illumination by a xenon lamp, showing only weak signals of $\mathrm{Fe}^{3+}$ and an unidentified center (the line at the magnetic field $\sim 3765 \mathrm{G}$ ). (c) EPR spectrum in the same sample after annealing at $1100{ }^{\circ} \mathrm{C}$ in $\mathrm{N}_{2}$ gas flow for 20 minutes measured in darkness showing a narrow signal of the shallow donor. 

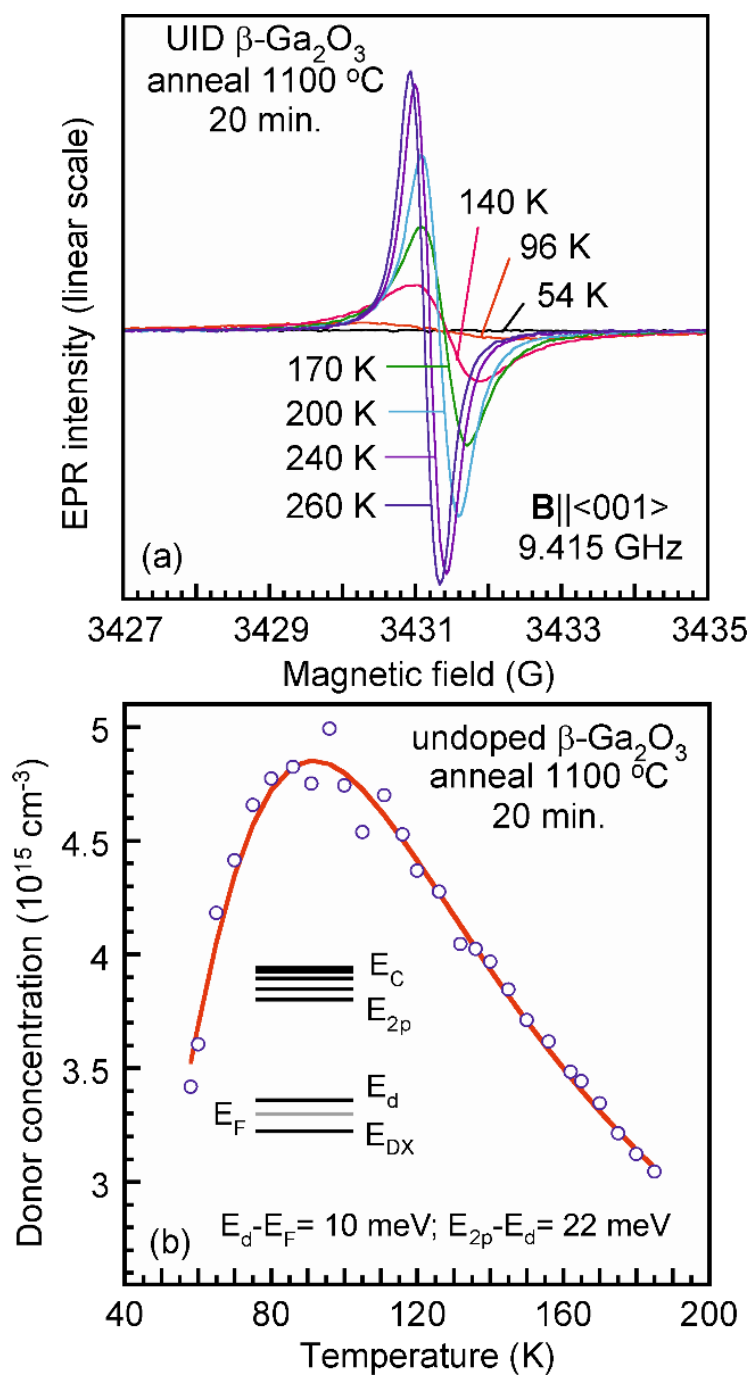

FIG. 2: (Color online) (a) EPR spectra of the residual shallow donor measured for $\mathbf{B} \|[001]$ at different temperatures in an UID $\beta-\mathrm{Ga}_{2} \mathrm{O}_{3}$ substrate annealed at $1100{ }^{\circ} \mathrm{C}$ in $\mathrm{N}_{2}$ gas flow for 20 minutes to partly activate the donor. All the spectra were measured with a field modulation of $0.4 \mathrm{G}$ and a microwave power of $0.6325 \mathrm{~mW}$. (b) The temperature dependence of the concentration of the donor in the neutral charge state determined from EPR. The solid curve represents the fit to the experimental data using Eq. (1). The inset shows the energy level scheme of a DX center. 


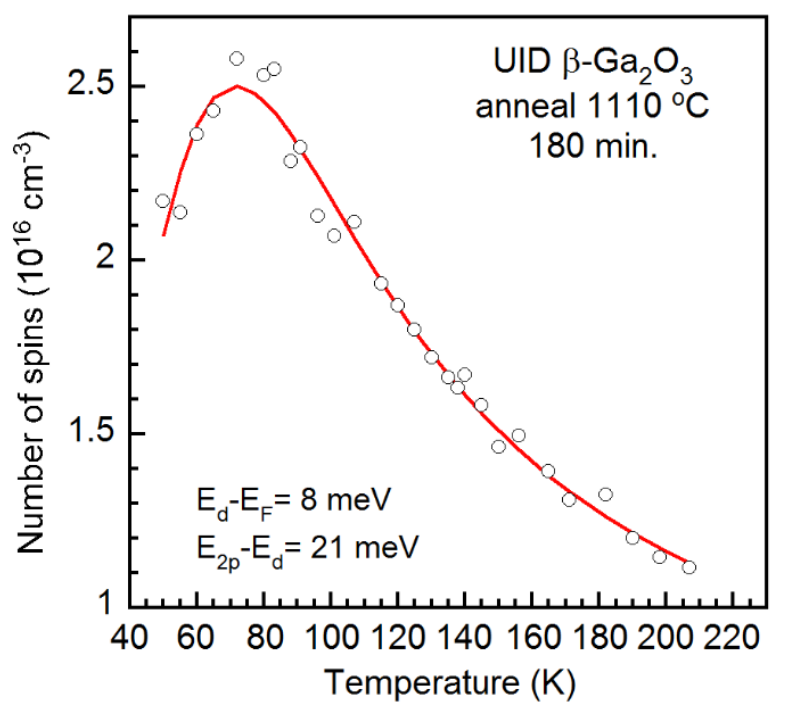

FIG. 3: (Color online) Temperature dependence of the concentration of the donor in the neutral charge state in the $\beta-\mathrm{Ga}_{2} \mathrm{O}_{3}$ sample mentioned in Fig. 2 after another anneal at 1110 ${ }^{\circ} \mathrm{C}$ for 180 minutes to further activate the residual donor. The solid curve represents the fit to the experimental data using Eq. (1).

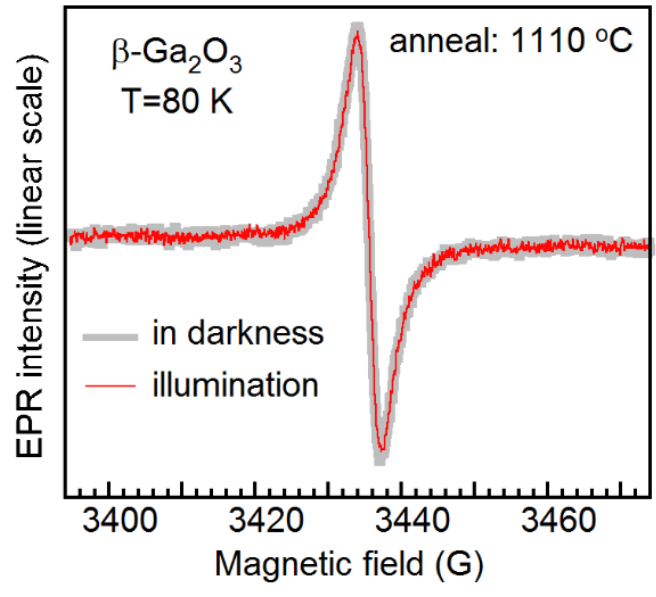

FIG. 4: (Color online) EPR spectra of the donor in the $\beta-\mathrm{Ga}_{2} \mathrm{O}_{3}$ sample mentioned in Fig. 3 measured for $\mathbf{B} \perp<001>$ (along the a-axis) at $80 \mathrm{~K}$ in darkness (thick grey line) and under illumination by a xenon lamp (thin red line), showing no effect of illumination on the donor signal. 


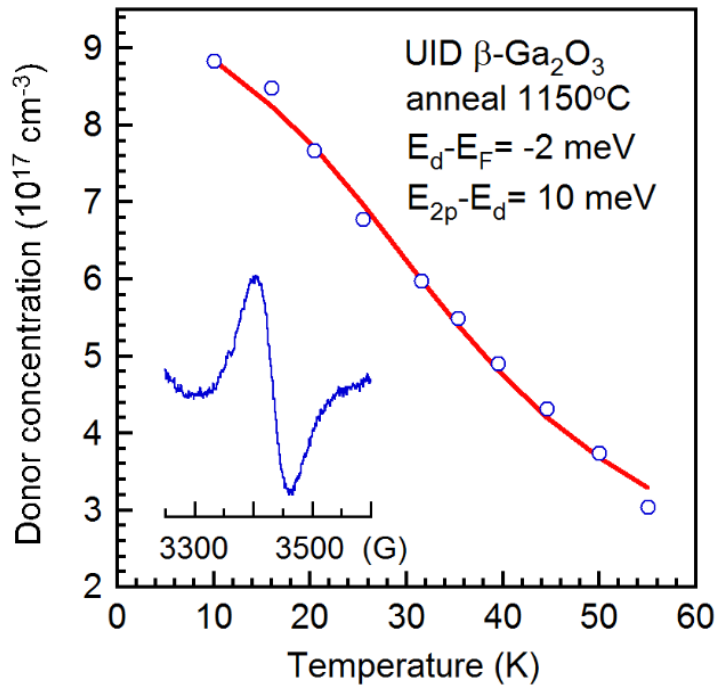

FIG. 5: (Color online) Temperature dependence of the unpaired spin density in an UID $\beta$ $\mathrm{Ga}_{2} \mathrm{O}_{3}$ sample fully activated of the residual donor by annealing at $1150{ }^{\circ} \mathrm{C}$ in $\mathrm{N}_{2}$ gas flow. The solid curve represents the fit to the experimental data using Eq. (2). The inset shows the EPR spectrum of the donor with a symmetric line shape. 


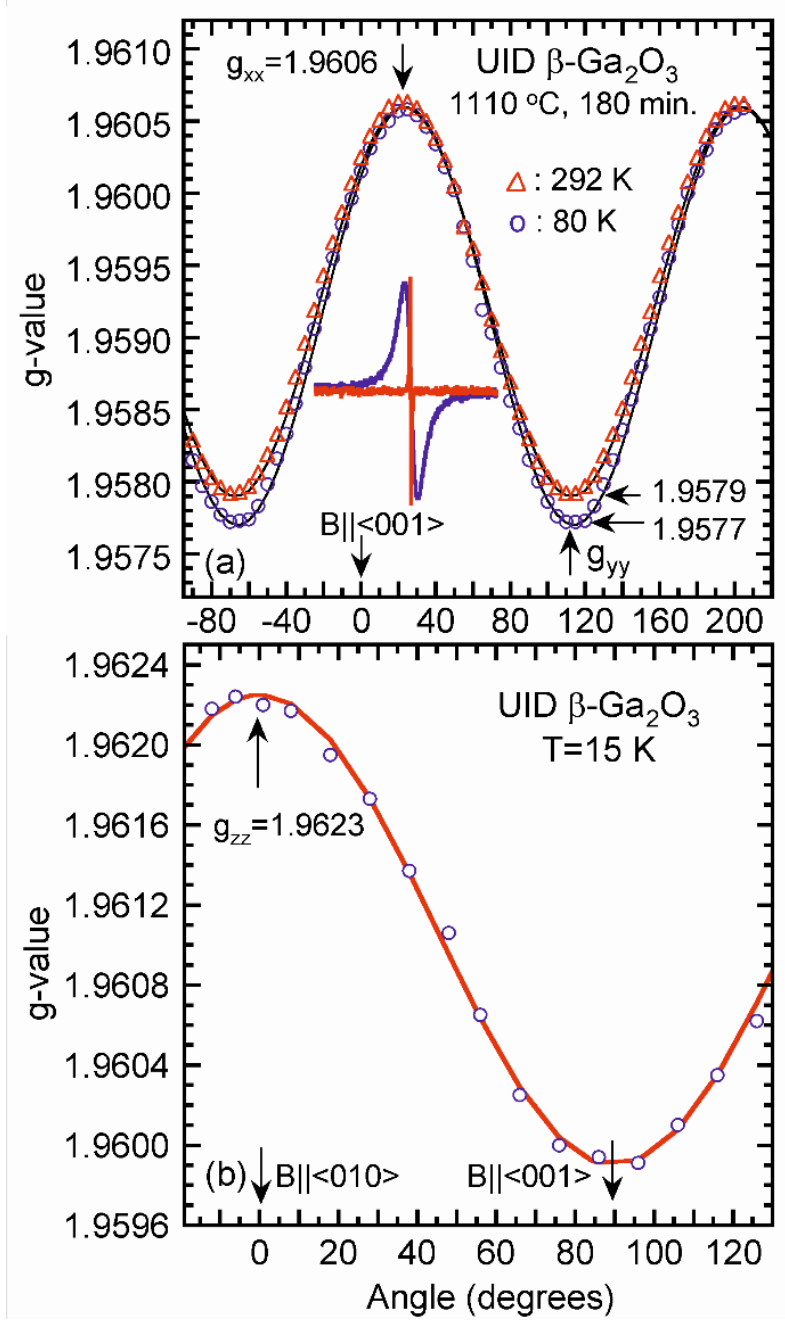

FIG. 6: (Color online) Angular dependence of the g-value of the donor spectra in UID $\beta$ $\mathrm{Ga}_{2} \mathrm{O}_{3}$ : (a) in the sample after annealing at and $1110{ }^{\circ} \mathrm{C}$ measured at $80 \mathrm{~K}$ (o) and at $292 \mathrm{~K}$ $(\Delta)$ with the magnetic field $\mathbf{B}$ rotating in the (010) plane; and (b) in a fully activated sample after annealing and $1150{ }^{\circ} \mathrm{C}$ measured at $15 \mathrm{~K}$ with $\mathbf{B}$ rotating from the $<010>$ direction to the $<001>$ direction. The solid curves represent the fitting of the experimental data. The inset in (a) shows narrow ( $\sim 0.22 \mathrm{G})$ and broad ( 3.7 G) EPR lines measured at $292 \mathrm{~K}$ and $80 \mathrm{~K}$, respectively. 


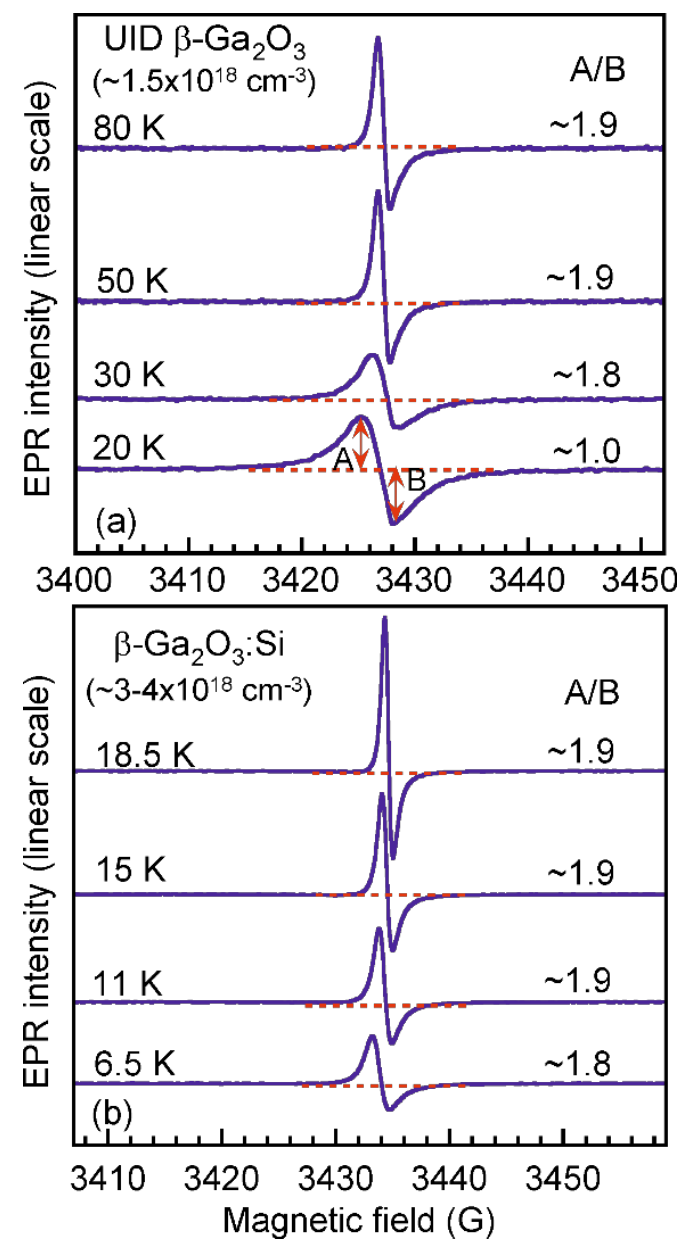

FIG. 7: (Color online) (a) EPR spectra in an UID, fully activated $\beta-\mathrm{Ga}_{2} \mathrm{O}_{3}$ sample with a spin density of $\sim 1.5 \times 10^{18} \mathrm{~cm}^{-3}$ showing the transition from Lorentzian line shape to Dysonian line shape caused by delocalized electrons at $\sim 30 \mathrm{~K}$. (b) In a (-201) Si-doped $\beta-\mathrm{Ga}_{2} \mathrm{O}_{3}$ sample with a higher donor concentration of $\sim 3-4 \times 10^{18} \mathrm{~cm}^{-3}$, the Dysonian line shape is already observed at $6.5 \mathrm{~K}$. With the same magnetic field scale of $52 \mathrm{G}$ in both (a) and (b), the linewidth of these spectra can be compared with each other directly. 


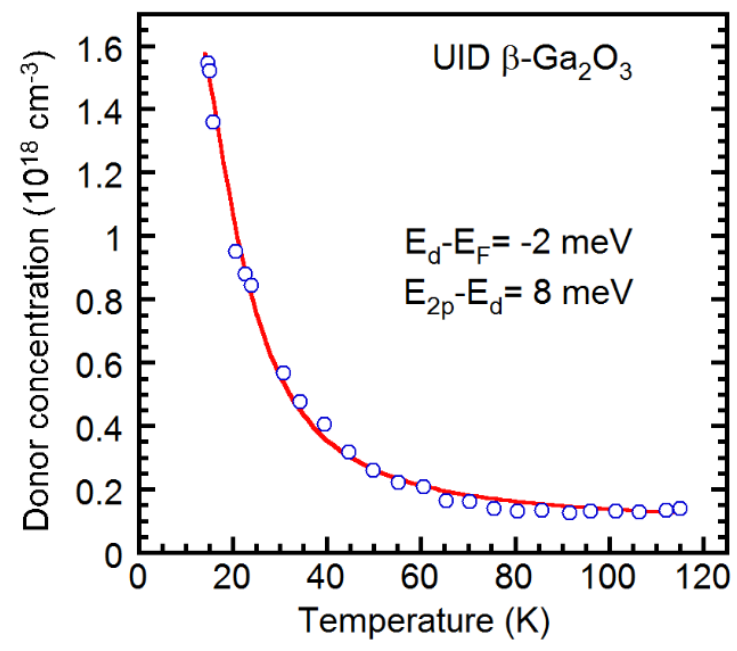

FIG. 8: (Color online) Temperature dependence of the unpaired spin density in the UID $\beta$ $\mathrm{Ga}_{2} \mathrm{O}_{3}$ sample mentioned in Fig. 7(a). The solid curve represents the fit to the experimental data using Eq. (2). 